\title{
Intrauterine Use of Autologous Platelet-Rich Plasma in Management of Asherman Syndrome: A Randomized Controlled Trial
}

\author{
Original \\ Article Mohamed Ibrahim Mohamed Amer, Reda Mokhtar Kamal Ghanem, Aya Sameh \\ AbdelRazek Oraby, Mohamed Abdellatif AbdelHaleem
}

Department of Obstetrics and Gynecology, Faculty of Medicine, Ain-Shams University, Egypt

\begin{abstract}
Background: Operative hysteroscopy with lysis of intrauterine adhesions is a viable treatment option for Asherman syndrome. However, with all forms of adhesion resection, there is a high rate of reformation of intrauterine adhesions. The current study will focus on a unique technique to evaluate the use of platelet rich plasma over an inflated balloon versus conventional method (inflated balloon).

Aim: This study aim to assess the efficacy of the platelet rich plasma in preventing reformation of intrauterine adhesions after adhesiolysis in cases of Asherman syndrome.

Materials and Methods: This study was conducted in Ain-Shams Maternity Hospital (Early Cancer Detection and Endoscopy Unit) during the period between July 2019 and February 2020. Forty patients seeking for conception with a history of primary or secondary infertility with severe intrauterine adhesions; 20 patients (case) injected with PRP and 20 patients (control) with IU balloon.

Results: Our study showed a significant increase of menses duration among the PRP group post-operative (3.0 \pm 1.1$)$ days and preoperative menses duration (1.5 \pm 1.4 ) days compared to balloon post-operative (1.9 \pm 1.6$)$ and preoperative (1.5 \pm 1.6$)$ days. The study also showed significant increase of menses amount among the PRP group post-operative (5.2 \pm 3.7$)$ pads, and preoperative menses duration (1.0 \pm 1.0$)$ pads compared to balloon post-operative $(2.9 \pm 3.2)$ and preoperative $(0.9 \pm 0.9)$ pads.

Conclusion: Platelet rich plasma after operative hysteroscopy has high efficacy and safety in improvement of menses duration, amount and adhesion score in cases suffering from severe intrauterine adhesions and decreasing postoperative adhesions.
\end{abstract}

Key Words: Asherman syndrome, infertility, platelet-rich plasma

Received: 15 April 2020, Accepted: 12 September 2020

Corresponding Author: Aya Sameh AbdelRazek Oraby, Department of Obstetrics and Gynaecology, Faculty of Medicine, Ain-Shams University, Egypt, Tel.: 01227051258, E-mail: aya090261@gmail.com

ISSN: 2090-7265, February 2021, Vol.11, No. 1

\section{INTRODUCTION}

Asherman syndrome (AS) is defined as intrauterine synechiae developed in response to injury to the pars basalis of the endometrium most commonly after uterine instrumentation associated with pregnancy ${ }^{[1]}$, such as curettage after miscarriage, postpartum bleeding, or retained placenta, as well as intrauterine surgery ${ }^{[2]}$. Tuberculosis is the most common infectious cause of intrauterine scarring worldwide, with poor response to treatment. The risk of intrauterine adhesions after one or two D\&C is about $14-16 \%$, which increases to $32 \%$ after the third D\&C. The severity of adhesions also increases with repeated $\mathrm{D} \& \mathrm{C}^{[3]}$.

The American Fertility Society classifies the severity of the disease in three stages as follow: mild disease; few filmy adhesions involving less than third of the uterine cavity with normal menses or hypomenorrhea. Moderate disease: filmy and dense adhesions, the involvement of one-third to two-thirds of cavity and hypomenorrhea and severe disease; dense adhesions involving more than twothirds of the cavity with amenorrhea.

The intrauterine adhesions can range from complete obliteration of the cavity to filmy, fluffy, or dense adhesions between uterine walls ${ }^{[4]}$. The main clinical presentations of the AS are infertility (43\%) and impairment of menstrual flow, from oligomenorrhea to amenorrhea (62\%). Hysteroscopy represents the gold standard method for the definitive diagnosis and treatment of the AS; the aim of treatment of AS is to restore a normal uterine cavity, resume normal menstruation and improve pregnancy outcomes ${ }^{[5]}$.

Successful hysteroscopic adhesiolysis may be performed using scissors or biopsy forceps, electrosurgery systems such as the monopolar cutting needle or versapoint bipolar, or using laser vaporization ${ }^{[6]}$. However, 
with all forms of adhesion resection, there is a high rate of reformation of intrauterine adhesions ranging between $3.1 \%$ and $23.5 \%$. The rate of reformation of adhesions is even higher in patients with severe adhesions $(20-62.5 \%)^{[7]}$.

Given this high rate of intrauterine adhesion reformation, many attempts have been made to decrease this rate. One unique technique was performed by Amer et al. ${ }^{[8]}$ that evaluated the use of a fresh amnion graft over an inflated balloon or a Foley catheter for 2 weeks at the time of hysteroscopic adhesiolysis in women with moderate and severe intrauterine adhesions. The authors noted a significant improvement in uterine length and intrauterine adhesions. This technique has made the suggestion that not only cutting the adhesions but also providing local stimulant factors to the endometrium would improve the outcome of treatment of $\mathrm{AS}^{[9]}$.

Platelet-rich plasma (PRP) is an autologous product derived from whole blood through the process of gradient density centrifugation. Autologous PRP has been shown to be safe and effective in promoting the natural processes of wound healing, soft tissue reconstruction, and bone reconstruction and augmentation ${ }^{[10]}$. PRP contains several growth factors such as vascular endothelial growth factor (VEGF), epidermal growth factor (EGF), platelet derived growth factor (PDGF), transforming growth factor (TGF) and other cytokines that stimulate proliferation and growth. Recently, PRP has been used in several medical conditions in ophthalmology, orthopaedics, surgery and wound healing ${ }^{[11]}$.

Intrauterine infusion of platelet-rich plasma (PRP) is a new approach that has been suggested for the treatment of thin endometrium ${ }^{[12]}$. Recently a case report of 2 patients treated by standard adhesiolysis of intrauterine adhesions and PRP infusion has shown treatment was well tolerated, with no short-term or long-term side effects, and appeared to improve endometrial function as demonstrated by successful conception and ongoing clinical pregnancies. In conjunction with solid in vitro data on human endometrial cells, these pilot clinical outcomes were very reassuring. There are no randomized controlled trials published in that field yet ${ }^{[13]}$.

\section{AIM OF THE WORK}

The aim of the study is to assess the efficacy of the platelet rich plasma in preventing reformation of intrauterine adhesions after adhesiolysis in cases of Asherman syndrome.

\section{PATIENTS AND METHODS}

Design: This is a prospective randomized controlled clinical trial.
Settings: Early cancer detection and endoscopy unit in the Gynaecology department at Ain-Shams University Hospital

Population: Forty women with AS were randomly distributed into two equal study and control groups.

Research Methodology: After approval of the ethical committee; women were enrolled in the study according to certain criteria.

Inclusion criteria: Age 18-38 years (peak of reproductive age). Severe intrauterine synechia confirmed by hysteroscopy ${ }^{[14]}$.

Exclusion criteria: Haemoglobin less than $10 \mathrm{~g} / \mathrm{dl}$; so the patient has a good reserve in case any complication happened. Platelet less than 150000/ $\mathrm{mm} 3$ to avoid intraoperative and postoperative bleeding tendency in an elective procedure. Patients taking anticoagulants to avoid intraoperative and postoperative bleeding and hematoma formation to decrease adhesion formation. Patients taking NSAID in the 15 days prior to the procedure to avoid malfunction of platelets. Active cervical or uterine infection, so as not to introduce infection during operation, any significant local association like large fibroid.

Randomization: Patients fulfilling criteria were randomized into two groups; allocation and concealment. The patients who are diagnosed previously with severe AS were chosen an envelope which determines the treatment method. There were 40 envelops assigning for both group mixed together in one box. This was ensured randomised allocation of patients to either group of this study through randomization tables. Hysteroscopic adhesiolysis was done to all participants in the study.

The study group included 20 patients. Adhesiolysis was followed by intrauterine use of PRP then insertion of intrauterine Foley balloon catheter that was inflated and left for 2 weeks. They were given antibiotics and hormonal treatment. Also, they were given low dose aspirin and sildenafil $50 \mathrm{mg}$ daily (Amer MI, Egyptian Journal, 2018)

The control group included 20 patients. Adhesiolysis was followed by insertion of intrauterine Foley balloon catheter that was inflated and left for 2 weeks. They were given antibiotics and hormonal treatment like the first group. Also, they were given low dose aspirin and sildenafil 50mg daily

Preparation of PRP: Thirty $\mathrm{ml}$ venous blood of one patient is put in a sterile tube with $1.25 \mathrm{ml}$ citrate phosphate dextrose adenine-1 (CPDA-1), mixed and agitated gently in a plain tube followed by 2 
step centrifugation: $1^{\text {st }}$ step (1000 gravitational force for 5 minutes); erythrocytes sediment but platelets remain in suspension. Upper plasma layer was transferred to another plain tube for condensation and centrifugation while the remaining lower cell layer was discarded. $2^{\text {nd }}$ step (1500 gravitational force for 15 minutes); supernatant layer (platelet- poor) was discarded and the lower $10 \mathrm{ml}$ (platelet-rich plasma) was preserved.

Fig.1: Sodium citrated tubes

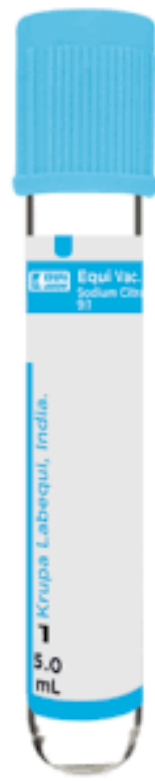

\section{All patients were subjected to the following:}

History taking; complete history was taken from patients that would include personal, menstrual, obstetric, medical and surgical history as well as history of any gynaecological problem and on-going treatments.

Examination: General, abdominal and pelvic examination

Hysteroscopic adhesiolysis: The procedure was done under general anaesthesia. The same hysteroscopy was used as before after adding an operative channel (model 26163V). Adhesions were removed by sharp scissors with saline distension medium or monopolar (Storz knife at power setting of $70 \mathrm{w}$ of pure cutting current and $40 \mathrm{w}$ of coagulation current with glycine as distension medium through an automated hysteroscopy distension pump. As mentioned above, PRP was divided into two equal samples. The liquid form was injected in the wall of the most affected zone via five inch flexible shielded injection needle (Egypt international company). Then Foley balloon catheter ( 8 French) was inflated inside the uterine cavity and was left for 2 weeks after cutting its stem. In the control group the balloon was inserted after the adhesiolysis immediately in the same way.
Patient was discharged as the same protocol of day cases and will be given the following postoperative medications: Oral hormonal therapy; $2 \mathrm{mg}$ Estradiol valerate for 21 days (Cycloprogynova, Bayer, Germany) (only white pills) for 3 months as well as low dose of aspirin and sildenafil $50 \mathrm{mg}$ daily.

Fig. 2: (a) Central adhesion. (b) Synechiolysis using scissors. (c) Marginal adhesion being lysed with bipolar twizzle. (d) Marginal adhesion being lysed with bipolar twizzle. (e) Cavity after lysis of right lateral synechia. The point of synechial detachment can be seen on the anterior wall. (f) Right fundo-cornual adhesion.

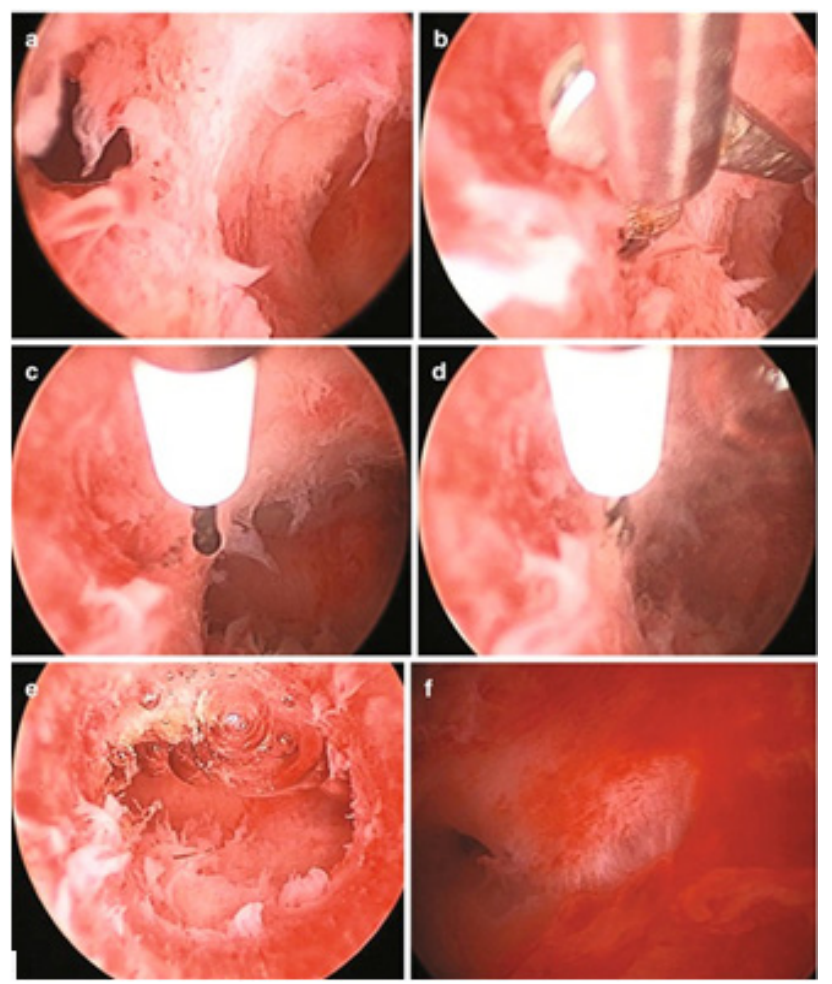

Primary outcome: Recurrence of adhesions. It was assessed and score was given according to the American fertility society ${ }^{[14]}$.

Secondary outcome: The pattern of menstrual flow. Persistence of amenorrhea or hypo-menorrhea or resumption of normal flow. This is assessed by number of days of flow and how many pads changed per day.

Sample size: Based on complete recovery in PRP, $60 \%$ compared to $20 \%$ in control group, alpha error $5 \%$, power of study $80 \%$, the required sample size is 40 patients 20 in each group. The program for sample size calculation is state 10 .

Ethics and Legal Data: The procedures set out in this study protocol pertaining to the conduct, evaluation and documentation of this proposed study are designed to ensure the researchers was followed the principles of Declaration of Helsinki in its current version laying down good clinical practice and ethical standards. 


\section{STATISTICAL ANALYSIS:}

The collected data were coded, tabulated, and statistically analyzed using IBM SPSS statistics (Statistical Package for Social Sciences) software version 22.0, IBM Corp., Chicago, USA, 2013. Descriptive statistics were done for quantitative data as minimum \& maximum of the range as well as mean $\pm \mathrm{SD}$ (standard deviation) for quantitative normally distributed data, while it was done for qualitative data as number and percentage. Inferential analyses were done for quantitative variables using Shapiro-Wilk test for normality testing, independent t-test in cases of two independent groups with normally distributed data and paired t-test in cases of two dependent groups with normally distributed data. In qualitative data, inferential analyses for independent variables was done using Chi square test for differences between proportions and Fisher's Exact test for variables with small expected numbers as well as McNemar test for agreement between paired categorical data. The level of significance taken at $P$ value $<0.050$ is significant, otherwise is non-significant. Intervention values were calculated as follows: Rate elevation=Study RateControl Rate. Efficacy= (Study Rate-Control Rate)/ Study rate. Relative Rate $=$ Study Rate/Control Rate Number needed to treat $=1 /($ Study Rate - Control Rate $)$.

\section{RESULTS}

No significant difference between the studied groups regarding demographic characteristics (Table 1).
Table 2 showed that grade-I/II adhesions was significantly more frequent among PRP group than among balloon group.

Table 3 showed that no significant difference between the studied groups regarding basal amenorrhea. Amenorrhea after intervention was non-significantly less frequent among PRP group than among balloon group.

Table 4 showed that no significant difference between the studied groups regarding last ordinary and before intervention menses duration then became significantly higher in PRP group. Gap between ordinary menses duration and menses duration after intervention was significantly lower in PRP group.

Elevation in menses duration after intervention as compared to that before intervention was significantly higher in PRP group. Menses duration was significantly increased in the studied group after intervention.

Table 5 showed that no significant difference between the studied groups regarding last ordinary and before intervention menses amount then became significantly higher in PRT group. Gap between ordinary menses amount and menses amount after intervention was significantly lower in PRT group. Elevation in menses amount after intervention as compared to that before intervention was significantly higher in PRT group. Menses amount was significantly increased in the studied group after intervention.

Table 1: Demographic characteristics among the studied groups

\begin{tabular}{|c|c|c|c|c|}
\hline Items & Measure & $\operatorname{PRP}(\mathrm{N}=20)$ & Baloon $(\mathrm{N}=20)$ & $P$ \\
\hline \multirow{3}{*}{ Age (years) } & Mean \pm SD & $32.8 \pm 3.7$ & $31.9 \pm 4.9$ & \multirow{3}{*}{$\wedge 0.515$} \\
\hline & & & & \\
\hline & Range & $23.0-38.0$ & $22.0-39.0$ & \\
\hline \multirow{3}{*}{ BMI (kg/m2) } & Mean \pm SD & $25.9 \pm 2.4$ & $26.1 \pm 2.1$ & \multirow{3}{*}{$\wedge 0.801$} \\
\hline & & & & \\
\hline & Range & $19.8-31.0$ & $22.8-29.8$ & \\
\hline \multirow{3}{*}{ Parity } & Nulligravida & $3(15.0 \%)$ & $2(10.0 \%)$ & \multirow{3}{*}{$\S 1.000$} \\
\hline & & & & \\
\hline & Multigravida & $17(85.0 \%)$ & $18(90.0 \%)$ & \\
\hline \multirow{5}{*}{ Possible etiology $(n, \%)$} & $\mathrm{CS}$ & $8(40.0 \%)$ & $8(40.0 \%)$ & \multirow{5}{*}{$\S 0.788$} \\
\hline & DandC & $8(40.0 \%)$ & $5(25.0 \%)$ & \\
\hline & Myomectomy & $1(5 \%)$ & $3(15 \%)$ & \\
\hline & TB & $1(5 \%)$ & $1(5 \%)$ & \\
\hline & Hysterotomy & $2(10 \%)$ & $3(15.0 \%)$ & \\
\hline
\end{tabular}

\footnotetext{
${ }^{\wedge}$ Independent t-test, §Fisher's Exact test
} 
Table 2: Adhesions grades among the studied groups

\begin{tabular}{|c|c|c|c|c|}
\hline Time & Grade & $\operatorname{PRP}(\mathrm{N}=20)$ & Baloon $(\mathrm{N}=20)$ & $\mathrm{P}$ \\
\hline Before & Grade-III/IV & $20(100.0 \%)$ & $20(100.0 \%)$ & -- \\
\hline After & Grade-I/II & $17(85.0 \%)$ & $11(55.0 \%)$ & $\# 0.038^{*}$ \\
\hline & Grade-III/IV & $3(15.0 \%)$ & $9(45.0 \%)$ & \\
\hline \multicolumn{5}{|c|}{ Value of PRP over balloon in getting grade-I/II } \\
\hline Items & & Value & \multicolumn{2}{|c|}{$95 \% \mathrm{CI}$} \\
\hline Rate elevation & & $30.0 \%$ & \multicolumn{2}{|c|}{$-3.4 \%-51.4 \%$} \\
\hline Efficacy & & $54.5 \%$ & \multicolumn{2}{|c|}{$-4.7 \%-100.0 \%$} \\
\hline Relative Rate & & 1.55 & \multicolumn{2}{|c|}{$0.95-2.16$} \\
\hline Number needed to treat & & 3.3 & \multicolumn{2}{|c|}{ 1.9- Infinite } \\
\hline
\end{tabular}

\#Chi square test, *significant

Table 3: Amenorrhea among the studied groups

\begin{tabular}{lccc}
\hline Time & PRP (N=20) & Balloon (N=20) & A -value (groups) \\
\hline Before & $8(40.0 \%)$ & $9(45.0 \%)$ & 0.749 \\
After & $1(5.0 \%)$ & $6(30.0 \%)$ & 0.091 \\
\#P-value (times) & $0.016^{*}$ & 0.250 & $95 \%$ CI \\
& Value of PRP over balloon in getting menses & $-3.5 \%-34.5 \%$ \\
Rate elevation & & Value & $-21.9 \%-99.2 \%$ \\
Efficacy & & $25.0 \%$ & $0.82-125.00$ \\
Relative Rate & & $83.3 \%$ & $2.9-$ Infinite \\
Number needed to treat & & 5.99 & 4.0 \\
\hline
\end{tabular}

${ }^{\wedge}$ Chi square test, \#McNemar test. *significant 
Table 4: Menses duration (days) among the studied groups

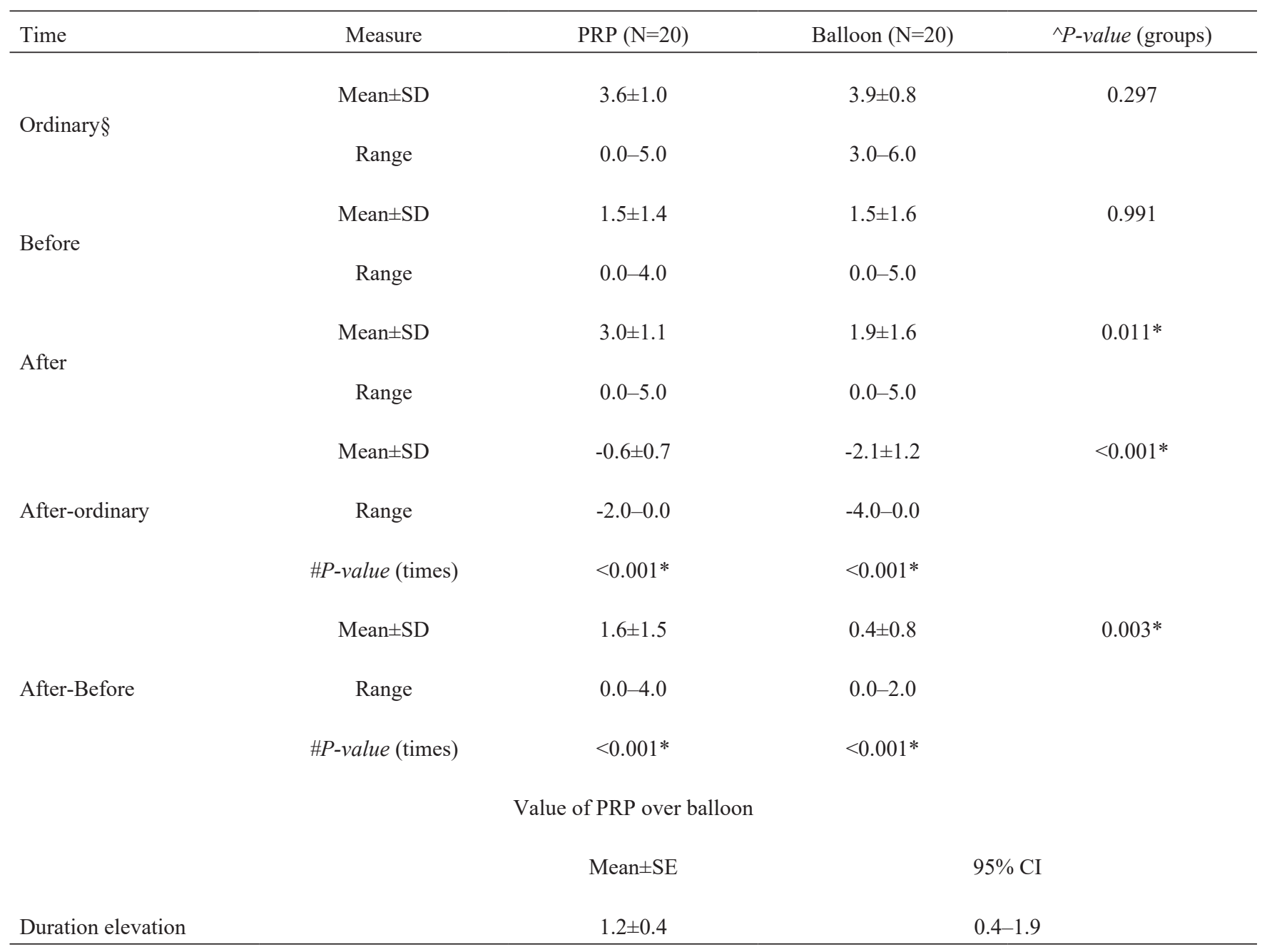

§Ordinary findings before adhesions, ^Independent t-test, \#Paired t-test, CI: Confidence interval, *Significant 
Table 5: Menses amount (pad) among the studied groups

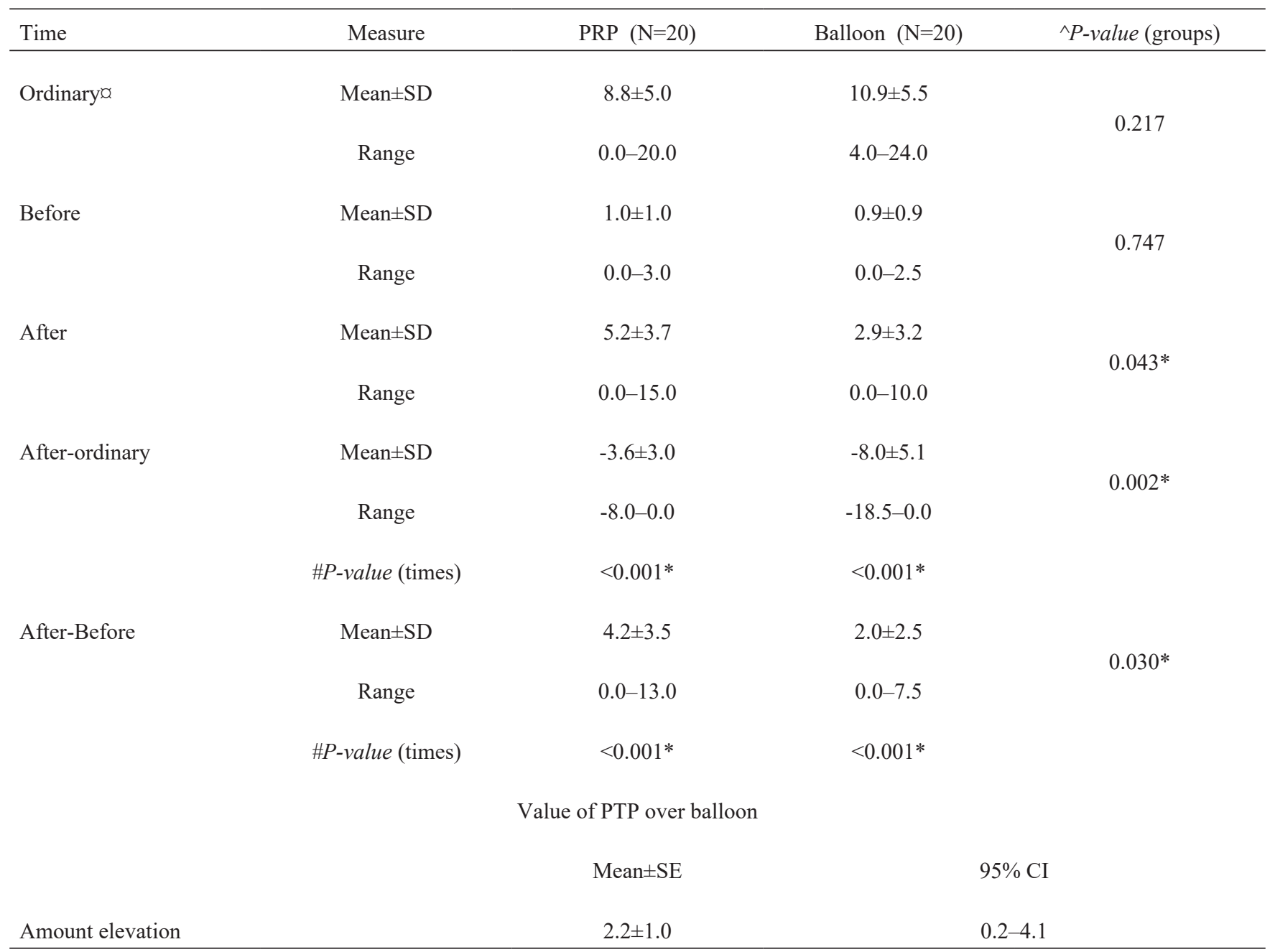

a Ordinary findings before adhesions, ^Independent t-test, \#Paired t-test, CI: Confidence interval, *Significant 


\section{DISCUSSION}

Intrauterine adhesions may be asymptomatic, but generally are related with infertility, menstrual disorders (hypomenorrhea, amenorrhea), cyclic pelvic pain or cramping around the time of period, recurrent miscarriage and endometriosis (caused by backflow of blood from the uterus). Indirect imaging methods give useful information in diagnosis as 3D ultrasound, but the gold standard diagnostic method is hysteroscopy which has the advantage of concurrent treatment ${ }^{[15]}$.

Management and treatment strategy include hysteroscopic adhesiolysis; readhesion prevention by intrauterine device, uterine balloon stent, Foley's catheter or amnion graft as well as restoration of normal endometrium (hormonal treatment-stem cells) and postoperative assessment by office hysteroscopy -ultrasound ${ }^{[2]}$.

Platelet rich plasma (PRP) is an autologous product derived from whole blood through the process of gradient density centrifugation. Autologous PRP has been shown to be safe and effective in promoting the natural processes of wound healing, soft tissue reconstruction, bone reconstruction and augmentation $^{[16]}$.

Recently, PRP has been used to promote endometrial growth and improve pregnancy outcome during in vitro fertilization in patients with persistent thin endometrium $(5-6 \mathrm{~m})^{[17]}$.

This current study was held to assess the efficacy of the use of plasma rich platelet versus IU balloon in decreasing recurrence of intrauterine adhesions after operative hysteroscopy.

We decided to design a prospective randomized controlled trial study at Ain-Shams Maternity Hospital (Early Cancer Detection Unit), from July 2019 to February 2020. Fifty-two patients were assessed for eligibility, eleven patients didn't meet the criteria and one patient refused to participate. The remaining 40 patients were randomized into two groups (20 patients in the study group underwent PRP injection and the 20 patients in the control group underwent balloon insertion).

\section{Patients fulfilling criteria will be randomized into two groups:}

Allocation and concealment: The patients who are diagnosed previously with severe AS will choose an envelope which determines the treatment method. There will be 40 envelops assigning for both group mixed together in one box. This will ensure randomised allocation of patients to either group of this study through randomization tables.

The study showed significant increase of menses duration among the PRP group post-operative (3.0 1.2$)$ days and preoperative menses duration $(1.4 \pm 1.5)$ days compared to balloon post-operative $(1.8 \pm 1.3)$ and preoperative $(1.3 \pm 1.4)$ days.

The study showed significant increase of menses amount among the PRP group post-operative (5.8 \pm 4.0$)$ pads, and preoperative menses duration $(1.0 \pm 1.0)$ pads compared to balloon post-operative $(2.9 \pm 2.7)$ and preoperative $(0.9 \pm 1.0)$ pads.

This study showed significant increase in the improvement of menses amount and duration in PRP group (93.3\%) and balloon group (80\%).

The study showed non-significant but higher improvement in adhesion score in the PRP. Before hysteroscopic adhesiolysis, 40 patients had grade 3 adhesions, 20 patients underwent PRP injection $[17 / 20(85 \%)$ patients became grade $(1,2)$ and $3 / 20(15 \%)$ patients became grade $(3,4)]$. Moreover, 20 patients underwent balloon [11/20 patients became grades $(1,2)(55 \%)$ and $9 / 20$ still have sever adhesions grade $(3,4)(45 \%)]$.

It is concluded that platelet rich plasma had high efficacy and safety in improvement of menses duration, amount and adhesion score in cases of severe intrauterine adhesions and decreasing postoperative adhesions.

Another parallel study showed that amniotic membrane increased menstrual blood volume after hysteroscopic adhesiolysis (mean difference 6.15, 95\% CI 4.20-8.11; $P<0.001)$. By contrast, this treatment did not affect the rates of intrauterine adhesion recurrence (RR 0.84, 95\% CI 0.61-1.16; $P=0.290$ ); pregnancy (RR 1.40, 95\% CI 0.78-2.50; $P=0.260$ ); or spontaneous abortion (RR 0.88, 95\% CI 0.38-1.99; $P=0.750)^{[18]}$.

Prevention of both formation and reformation of intrauterine adhesions is a challenging issue in clinical practice. A variety of methods have been proposed including hormone treatment, intrauterine mechanical barriers, such as IUD s and intrauterine balloons, which all appear to have some benefit. Both Hyaluronic acid gel and amnion graft have promising results but further clinical trials are required to confirm their efficacy ${ }^{[8,19]}$.

Another study showed that placement of intrauterine freeze-dried AM graft following operative 
hysteroscopic procedure was associated with significant improvement regarding uterine length, duration of menses and risk of recurrence of moderate/ severe IUA.

In a previous two published studies, Amer et al. used fresh AM grafts after hysteroscopic adhesiolysis. In the first non-randomized pilot study, Amer and Abd-El-Maeboud compared postoperative placement of intrauterine AM graft $(n=12)$ versus placement of intrauterine inflated balloon of a Foley's catheter $(n=13)$. The rates of amenorrhea 4 months postoperatively were $16.7 \%$ and $23.1 \%$, respectively. The rates of recurrent adhesions (though minimal in most cases) were $0 \%$ and $100 \%$ in both groups, respectively ${ }^{[8]}$

In the second randomized controlled study, Amer et al. compared intrauterine placement of inflated balloon of a Foley's catheter $(n=15)$ versus placement of fresh AM graft $(n=15)$ versus placement of dried AM graft $(n=15)$. The rates of restoration of normal menses were $26.8 \%, 35.7 \%$ and $46.7 \%$, respectively. The rates of pregnancy were $80 \%$ and $20 \%$ in women who had AM graft and those who did not, respectively ${ }^{[20]}$.

Freeze-drying of AM graft offers three advantages: proper disinfection, long-term preservation and maintenance of structural integrity of the AM graft, which allows for adequate re-epithelialization and restoration of normal endometrial lining.

When compared to other alternatives, placement AM graft after hysteroscopic adhesiolysis seems to be associated with better outcomes. In a study conducted by Lin et al. placement of intrauterine heart-shaped balloon $(n=82)$ was compared to placement of intrauterine device $(n=80)$ following hysteroscopic adhesiolysis, the rates of adhesion reformation after $1-2$ months were $30 \%$ and $35 \%$, respectively ${ }^{[21]}$.

A prospective, randomized, controlled study was performed to assess the efficacy of auto-crosslinked hyaluronic acid (ACP) gel in preventing the development of intrauterine adhesions following hysteroscopic adhesiolysis. Ninety-two patients with irregular menses and intrauterine adhesions referred to the Hysteroscopic Unit of the University of Naples "Federico II". Patients were randomized to two different groups. Group A were randomized to hysteroscopic adhesiolysis plus intrauterine application of ACP gel $(10 \mathrm{ml})$ and group B were randomized to operative hysteroscopy alone (control group). Baseline adhesion scores were calculated for each patient and at 3 months after surgery. Group A showed a significant decrease in intrauterine adhesions at 3 months follow- up in comparison with the control group. Staging of adhesions showed a significant decrease in adhesion severity in patients treated with ACP gel ${ }^{[22]}$.

In a small nonrandomized study, postoperative IUD plus hormone therapy was compared with hormone therapy alone; no significant difference was found insofar as re-formation of adhesions. The risk of infection when an IUD is introduced into the uterus immediately after adhesiolysis is estimated to be $8 \%$, and perforation of the uterus during IUD insertion has been reported. The use of a Foley catheter for 3 to 10 days after surgical lysis of IUAs is similarly reported to act as a physical intrauterine barrier ${ }^{[20]}$.

A non randomized study compared use of an inflated pediatric Foley catheter in place for 10 days postoperatively in 59 patients with that of an IUD in situ for 3 months in 51 patients. There were fewer infections in the Foley group and a lower recurrence rate of IUAs as assessed using HSG. Although amenorrhea continued in $19 \%$ of women in the Foley group and $38 \%$ in the IUD group, the fertility rate was relatively low in both groups: 20 of $59(34 \%)$ and 14 of $51(28 \%)$, respectively. In a study of 25 women with moderate to severe IUAs, use of a fresh amnion graft over an inflated Foley catheter prevented recurrence of IUAs in $52 \%$ of women, although follow-up fertility data and complications are not reported ${ }^{[20]}$.

A three-armed pilot RCT assessed fresh amnion versus dried amnion grafts versus intrauterine balloon alone $^{[20]}$.

Forty-five women were randomized (15 in each group) and each underwent diagnostic hysteroscopy 2 to 4 months following treatment. Amnion grafts reduced adhesions significantly more than balloon alone (p.003) and fresh amnion was superior to dried amnion (p.05). Ten women (23\%) conceived with $6(60 \%)$ having a miscarriage. The issue of infection with the insertion of an intrauterine stent has been assessed in an RCT of 60 women (30 women randomized to receive the stent; 30 women as a control) $)^{[21]}$.

Hysteroscopic procedures were performed and the outcome measure was bacterial colonization 30 days after the procedure. There was no difference between control $(13 \%$ and $33 \%)$ and stent $(10 \%$ and $30 \%$ ) for bacterial colonization rates before and after stent placement suggesting that infection risk is not substantially impacted by the use of an intrauterine stent $^{[23]}$.

A number of newer adhesion barriers are modifications of hyaluronic acid that have been 
reported to be successful after treatment of IUAs. There is one class 1 study of 150 women who underwent suction curettage after incomplete, missed, or recurrent miscarriage. Fifty women were randomized to receive an adhesion barrier (Seprafilm; Genzyme Corp., Cambridge Massachusetts), and 100 patients served as the control group. In the adhesion barrier group, 32 of 32 patients $(100 \%)$ became pregnant in the 8 months after the procedure compared with 34 of 56 patients $(54 \%)$ in the control group. Adhesions were found in 1 of 10 women $(10 \%)$ women receiving treatment compared with 7 of $14(50 \%)$ in the control group who had not become pregnant. No adverse events were reported in the treatment group. Autocross-linked hyaluronic acid gel may also be suitable for preventing IUAs because of high sensitivity and prolonged residency time on an injured surface. In a randomized control trial of 84 women, auto-crosslinked hyaluronic acid gel (Hyalobarrier gel; FABFidia Advanced Polymers, Abano-Terme, Italy) was compared with no therapy after surgical treatment of Asherman syndrome. Postoperative ultrasound studies demonstrated that the walls of the uterine cavity remained separated for at least 72 hours. At secondlook hysteroscopy 3 months after the procedure, IUAs were substantially reduced in patients receiving the adhesion barrier compared with the control group (6 of $43[14 \%]$ vs13 of 41 [32\%); p.05) ${ }^{[23]}$.

Stem cell treatments for intrauterine adhesions. The use of human stem cell treatments for the reconstruction of the endometrium following substantial damage and IUA formation has been hypothesized for some time, with studies from animal models showing substantial promise in this area of medical treatment. From the first prospective series in humans, 16 women with substantial hysteroscopically confirmed IUAs were treated by uterine intravascular infusions of BMDSC. Clinical, hysteroscopic, and fertility data are reported subsequently, with menstrual function returning to normal within six months of BMDSC infusion and three spontaneous pregnancies and seven pregnancies following in vitro fertilization recorded. These initial data from a human series represent the first adjunct treatment of this type for the treatment of Asherman syndrome with successful menstrual and fertility outcomes. It is imperative that well-conducted RCTs are performed to establish the role of BMDSC treatment in addition to or independent of surgical treatments before it is made ${ }^{[24]}$.

Hormonal treatments postoperative treatment with estrogen therapy (a daily oral dose of $2.5 \mathrm{mg}$ conjugated equine estrogen with or without opposing progestin for 2 or 3 cycles) has been described after surgical treatment of intrauterine adhesions. No comparative studies have been performed investigating dosage, administration, or combination of hormones. One nonrandomized study reported that hormone treatment alone is as effective as hormone treatment and IUD in combination. Techniques to Increase Vascular Flow to Endometrium Various studies have described use of medications such as aspirin, nitroglycerine, and sildenafil citrate to increase vascular perfusion to the endometrium and enable pregnancy ${ }^{[24]}$.

Antibiotic therapy, there are no data to support the use of antibiotic therapy before, during, or after surgical treatment of Asherman syndrome. The American College of Obstetricians and Gynecologists guidelines for antibiotic use in gynecologic procedures do not recommend ${ }^{[24]}$.

\section{CONCLUSION}

Platelet rich plasma after operative hysteroscopy has high efficacy and safety in improvement of menses duration, amount and adhesion score in cases suffering from severe intrauterine adhesions and decreasing postoperative adhesions.

\section{CONFLICT OF INTEREST}

There are no conflicts of interests.

\section{REFERENCES}

1. Cenksoy PO, Ficicioglu C, Yesiladali M, Kizilkale O. The diagnosis and management of Asherman's syndrome developed after cesarean section and reproductive outcome. Case reports in obstetrics and gynecology. 2013; 450658; doi: $10.1155 / 2013 / 450658$.

2. Conforti A, Alviggi C, Mollo A, De Placido G and Magos A. The management of Asherman syndrome: a review of literature: Reproductive Biology and Endocrinology, 2013; 11(1):118.

3. Grace GA, Devaleenal DB, Natrajan M. Genital tuberculosis in females. The Indian journal of medical research. 2017; 145(4):425.

4. Aghajanova L, Cedars MI, Huddleston HG. Platelet-rich plasma in the management of Asherman syndrome: case report. Journal of assisted reproduction and genetics. 2018; 35(5):771-5.

5. Pop-Trajković-Dinić S, Ljubić A, Kopitović V, Antić V, Stamenović S, Trninić-Pjević A. The 
role of hysteroscopy in diagnosis and treatment of postmenopausal bleeding. Vojnosanitetski pregled. 2013; 70(8):747-50.

6. Salma U, Xue M, Sayed M, Sheikh A, Xu D. Efficacy of intrauterine device in the treatment of intrauterine adhesions. BioMed research international. 2014; doi: 10.1155/2014/ 589296

7. Gambadauro $\mathrm{P}$, Gudmundsson $\mathrm{J}$, Torrejón R. Intrauterine adhesions following conservative treatment of uterine fibroids. Obstetrics and gynecology international. 2012; doi: $10.1155 / 2012 / 853269$.

8. Amer MI and Abd-El-Maeboud KH. Amnion graft following hysteroscopic lysis of intra uterine adhesions, J Obstet Gynecol Research. 2006; 32(8): 559-566.

9. Peng X, Li T, Zhao Y, Guo Y, Xia E. Safety and efficacy of amnion graft in preventing reformation of intrauterine adhesions. Journal of minimally invasive gynecology. 2017; 24(7):1204-10.

10. Dhurat R and Sukesh MS. (2014): Principles and methods of preparation of platelet-rich plasma: a review and author's perspective. Journal of cutaneous and aesthetic surgery; 7(4):189.

11. Lee JW, Kwon OH, Kim TK, Cho YK, Choi KY, Chung HY, Cho BC, Yang JD, Shin JH. Plateletrich plasma: quantitative assessment of growth factor levels and comparative analysis of activated and inactivated groups. Archives of plastic surgery. 2013; 40(5):530.

12. Nazari L, Salehpour S, Hoseini S, Zadehmodarres $\mathrm{S}$, Ajori L. Effects of autologous platelet-rich plasma on implantation and pregnancy in repeated implantation failure: a pilot study. International Journal of Reproductive BioMedicine. 2016; 14(10):625.

13. Zadehmodarres S, Salehpour S, Saharkhiz N, Nazari L. Treatment of thin endometrium with autologous platelet-rich plasma: a pilot study. JBRA assisted reproduction. 2017; 21(1):54.
14. The American Fertility Society: Classification of adnexal adhesions, distal tubal occlusion, tubal occlusion secondary to tubal ligation, tubal pregnancies, Mullerian anomalies and intrauterine adhesions. Fertil.Steril 1988; 49 :944-955.

15. Sendag F, Dikmen A, Akman L and Oztekin $\mathrm{K}$. Hysteroscopic management of intrauterine adhesion: Journal of Turkish Society of Obstetrics and Gynecology,2013;10(1):21-25.

16. Smith RG, Gassmann CJ and Campbell MS. Platelet-rich plasma: properties and clinical applications: J Lancaster Gen Hosp, 2007; 2(2):73-8.

17. Chang Y, Li J, Chen Y, Wei L, Yang X, Shi Y and Liang $\mathrm{X}$. Autologous platelet-rich plasma promotes endometrial growth and improves pregnancy outcome during in vitro fertilization: International Journal of Clinical and Experimental Medicine, 2015; 8(1):1286.

18. Zheng F, Zhu B, Liu Y, Wang R, Cui Y. Metaanalysis of the use of amniotic membrane to prevent recurrence of intrauterine adhesion after hysteroscopic adhesiolysis. 2018.

19. Deans R and Abbott J. Review of intrauterine adhesions. J Minim Invasive Gynecol. 2010; 11:555-569.

20. Amer MI, Abd-El-Maeboud KH, Abdelfatah I, Salama FA. Human amnion as a temporary biologic barrier after hysteroscopic lysis of severe intrauterine adhesions: pilot study. Journal of minimally invasive gynecology. 2010; 17(5):605-11.

21. Lin XN, Zhou F, Wei ML, Yang Y, Li Y, Li TC, Zhang SY. Randomized, controlled trial comparing the efficacy of intrauterine balloon and intrauterine contraceptive device in the prevention of adhesion reformation after hysteroscopic adhesiolysis. Fertility and sterility. 2015; 104(1):235-40.

22. Acunzo G, Guida M, Pellicano M, Tommaselli GA, Di Spiezio Sardo A, Bifulco G, Cirillo D, Taylor 
A, Nappi C. Effectiveness of auto-cross-linked hyaluronic acid gel in the prevention of intrauterine adhesions after hysteroscopic adhesiolysis: a prospective, randomized, controlled study. Human reproduction. 2003; 18(9):1918-21.

23. Mazzon I, Favilli A, Cocco P, et al. Does cold loop hysteroscopic myomectomy reduce intrauterine adhesions? A retrospective study. Fertil Steril. 2014; 101:294-298.

24. Pabuccu R, Atay V, Orhon E, Urman B, Erg€un A. Hysteroscopic treatment of intrauterine adhesions is safe and effective in the restoration of normal menstruation and fertility. Fertil Steril. 1997; 68:1141-1143. 